\title{
Prevalence of Autonomic Dysreflexia in Patients with Spinal Cord Injury above T6
}

\author{
Eun Sun Lee ${ }^{1}$ and Min Cheol Joo ${ }^{2}$ \\ ${ }^{1}$ Department of Rehabilitation Medicine, Wonkwang University School of Medicine, 895 Muwang-ro, Iksan, \\ Jeonbuk 54538, Republic of Korea \\ ${ }^{2}$ Department of Rehabilitation Medicine and Institute of Wonkwang Medical Science, Wonkwang University School of Medicine, \\ 895 Muwang-ro, Iksan, Jeonbuk 54538, Republic of Korea \\ Correspondence should be addressed to Min Cheol Joo; jmc77@daum.net
}

Received 21 July 2017; Accepted 3 October 2017; Published 26 October 2017

Academic Editor: Antonio Salgado

Copyright (C) 2017 Eun Sun Lee and Min Cheol Joo. This is an open access article distributed under the Creative Commons Attribution License, which permits unrestricted use, distribution, and reproduction in any medium, provided the original work is properly cited.

\begin{abstract}
Objective. To investigate the prevalence of autonomic dysreflexia (AD) using ambulatory blood pressure monitoring (ABPM) and the autonomic dysfunction following spinal cord injury (ADFSCI) questionnaire in patients with spinal cord injury (SCI) above T6. Methods. Twenty-eight patients diagnosed with SCI above T6 were enrolled. ABPM and ADFSCI were utilized to assess AD. Using $\mathrm{ABPM}$, systolic blood pressure (SBP), diastolic blood pressure, and heart rate (HR) were measured at 30-minute intervals. AD was defined as SBP $20 \mathrm{mmHg}$ higher than basal SBP, and the number of AD events was counted. The ADFSCI questionnaire evaluates the severity and frequency of the AD symptoms. Results. According to the ABPM, AD occurred in 26 patients and AD events occurred $5.8 \pm 4.7$ times. Average daytime and nighttime SBP were $119.9 \pm 18.8 \mathrm{mmHg}$ and $123.8 \pm 21.2 \mathrm{mmHg}$, respectively, and the nighttime mean SBP appeared to be $4 \mathrm{mmHg}$ higher than daytime mean SBP. These findings suggest the loss of nocturnal BP dipping in SCI patients. ADFSCI results revealed that 16 of the patients evaluated were symptomatic while 12 were asymptomatic. Conclusion. AD following SCI above T6 was highly prevalent and several patients seemed asymptomatic. These results suggest the necessity of proper diagnostic and therapeutic interventions for managing AD.
\end{abstract}

\section{Introduction}

Autonomic dysreflexia (AD) is a disease that commonly occurs in patients with spinal cord injury (SCI), especially in the cervical and upper thoracic level above T6 $[1,2]$. It is an acute disease with symptoms ranging from mild, such as headache, sweating, hot flashes, piloerection, and anxiety, to severe [3-7], such as arrhythmia, including atrial fibrillation, and high systolic blood pressure (SBP) above $300 \mathrm{mmHg}$, which may lead not only to cerebral hemorrhage but also to convulsions and death [8-10].

Cardiovascular disease is one of the most common causes of death in patients with SCI. Moreover, the underlying causes of cardiovascular disease are due to abnormal blood pressure (BP) control such as AD and orthostatic hypotension (OH). A high prevalence of arrhythmia and abnormal cardiovascular responses after exercise are also important causes of cardiovascular disease [11]. In general, the diagnostic definition of $\mathrm{AD}$ is a rise in SBP by more than $20-40 \mathrm{mmHg}$ [5]. However, considering that the baseline $\mathrm{BP}$ of patients with cervical or upper thoracic SCI has been reported to be lower than that of healthy persons by $15-20 \mathrm{mmHg}[6,7]$, normal or slightly elevated $\mathrm{BP}$ can be diagnosed as $\mathrm{AD}$ in patients with SCI. But one-time assessments, such as individual BP measurement, which are clinically practiced currently, or diagnosis depending on patients' symptoms, are not sufficient to make an accurate diagnosis of the various aspects of BP instability earlier in patients with SCI. Accordingly, a recent report proposing a method to assess such instability through long-term and real-time measurements of BP in patients with SCI using circadian BP profiles and ambulatory blood pressure monitoring (ABPM) has been shown to 
possess a good prognostic value for cardiovascular morbidity and mortality [12]. ABPM is an automated, noninvasive assessment method, which is useful for $\mathrm{AD}$ diagnosis and circadian BP assessment by continuous measurements of BP and heart rate (HR) during a period of 24 hours.

Therefore, in this study, we use the ABPM in patients with cervical or upper thoracic SCI above T6 in order to estimate the prevalence of $\mathrm{AD}$ by examining its incidence rate and patterns. Furthermore, the incidence rate of symptomatic or asymptomatic $\mathrm{AD}$ was examined using the autonomic dysfunction following spinal cord injury (ADFSCI) questionnaire [13] to propose the need for early diagnosis and interventions for asymptomatic AD.

\section{Methods}

2.1. Subjects. The subjects of this study were patients with SCI who were admitted to the rehabilitation department of Wonkwang University Hospital. The inclusion criteria for the subjects were as follows: (i) patients diagnosed with cervical or upper thoracic SCI above T6 via medical imaging or clinical assessment; (ii) patient age at least 19 years; (iii) patients without complications such as pneumonia and urinary tract infections; and (iv) neurologically stable patients who consented to participate in this study. Patients with serious or unstable physical diseases or patients with a past medical history of severe cardiovascular disease except hypertension were excluded.

The medical record of each patient including the demographic information (age, sex, cause of SCI, duration of SCI, etc.) was recorded and ABPM and ADFSCI questionnaire were administered. Neurological levels and SCI severity were determined using the American Spinal Injury Association Impairment Scale (AIS).

All studies were carried out in accordance with relevant standards after obtaining the approval of the Institutional Review Board (IRB number: 201601-HR-004) of Wonkwang University Hospital, and patients signed a written consent form prior to study participation after being informed about the purpose, methods, and other relevant information regarding the study.

\subsection{Methods}

2.2.1. Functional Evaluation of the Autonomic Nervous System. For ABPM, a BR 102 plus (Schiller-Reomed AG, Baar, Zug, Switzerland) was attached to the nondominant upper limb of the patient using a cuff of an appropriate size, and SBP, diastolic BP (DBP), and HR were recorded within a 24hour period. Collected data were analyzed using the MT300 software. The 12-hour period between 7:00 a.m. and 7:00 p.m. was designated as daytime and the remaining time was designated as nighttime. SBP, DBP, and HR were measured every 30 minutes. Additionally, the patients were instructed to press the button for self-monitoring of $\mathrm{BP}$ whenever $\mathrm{AD}$ symptoms appeared.

$\mathrm{AD}$ was defined as SBP exceeding the baseline BP by $20 \mathrm{mmHg}$ [2] and the baseline BP was obtained by calculating the averages of SBP measured during the 12 hours both in the daytime and in the nighttime. The frequency of AD was recorded as the number of incidences during the daytime and nighttime and also as the sum of daytime and nighttime incidences.

2.2.2. Questionnaire of Autonomic Dysfunction following Spinal Cord Injury. To evaluate AD symptoms, AD criteria in Part III of the ADFSCI were used [13]. The evaluation criteria were the presence or absence and frequency of the following symptoms, which included headache, excessive sweating in the body above the area of SCI, goosebumps or anxiety, and palpitations. These were evaluated using a five-point scale system according to the degree of symptom severity ( 0 : not at all; 5: very often, very severe) with a range of $0 \sim 204$ points, and through this method, the frequency and severity of $\mathrm{AD}$ symptoms were recorded.

2.2.3. Statistical Analysis. For statistical analysis, SPSS ver. 19.4 (IBM SPSS, Armonk, NY, USA) was used and the statistical significance was set at $p<0.05$. Differences in the results of ABPM between daytime and nighttime were analyzed using the paired $t$-test and intergroup differences depending on the duration of symptoms and the presence or absence of $\mathrm{AD}$ symptoms were analyzed using the independent $t$-test.

\section{Results}

3.1. General Characteristics of the Subjects. The study included 22 men and 6 women with a mean age of $56.8 \pm 10.5$ (range: 31-77) years. Regarding the cause of SCI, 8 and 20 patients had SCI due to disease and trauma, respectively. SCI duration ranged from 7 days to 31 years and 7 months with an average duration of $28.4 \pm 76.6$ months. As for the distribution by duration of SCI, less than 3 months and longer than 3 months after injury were defined as the subacute stage and the chronic stage. There were 15 and 13 patients in the subacute and chronic groups, respectively. Regarding the level of injury, 24 patients had cervical SCI and 4 patients had thoracic SCI. With respect to SCI severity, 4 patients were classified as having motor complete SCI (AIS grades A and B) and 24 patients were classified as having motor incomplete SCI (AIS grades C and D) (Table 1).

3.2. Results of $A B P M$. The 24-hour SBP, DBP, and HR for all subjects were $120.6 \pm 18.9 \mathrm{mmHg}, 75.8 \pm 10.7 \mathrm{mmHg}$, and $75.1 \pm 13.4 \mathrm{bpm}$, respectively. The daytime SBP, DBP, and HR were $119.8 \pm 18.8 \mathrm{mmHg}, 75.6 \pm 11.0 \mathrm{mmHg}$, and $76.3 \pm$ $14.1 \mathrm{bpm}$, respectively, and the nighttime SBP, DBP, and HR were $123.8 \pm 21.2 \mathrm{mmHg}, 77.4 \pm 11.1 \mathrm{mmHg}$, and $71.2 \pm$ $13.1 \mathrm{bpm}$, respectively. It was observed that the nighttime SBP and DBP were higher than the daytime values, showing the loss of physiologic nocturnal dipping, and the nighttime HR was statistically significantly lower $(p<0.05)$. Twentysix of the 28 patients (92.8\%) had AD at least once and the average $\mathrm{AD}$ frequency was $2.7 \pm 1.9$ times during daytime and $3.1 \pm 4.8$ times during nighttime. The average $\mathrm{AD}$ frequency in 24 hours was $5.8 \pm 4.7$ times (Table 2 ). General characteristics of $\mathrm{AD}$ and non-AD groups were age (56.0 versus 61.0 ), male sex $(75 \%$ versus $100 \%)$, cause of SCI (trauma $=66.7 \%$ versus 
TABLE 1: General characteristics of patients with spinal cord injury $(n=28)$

\begin{tabular}{lc}
\hline Demographic factor & Value \\
\hline Gender (male : female) & $22: 6$ \\
Mean age (yr) & $56.8 \pm 10.5$ \\
Etiology (spontaneous : traumatic) & $8: 20$ \\
Lesion duration (mo) & $28.4 \pm 76.6$ \\
$\quad$ Subacute & 15 \\
$\quad$ Chronic & 13 \\
Level of spinal cord injury & \\
$\quad$ Cervical & 24 \\
$\quad$ Thoracic & 4 \\
ASIA scale & \\
$\quad$ Motor complete (grades A and B) & 4 \\
$\quad$ Motor incomplete (grades C and D) & 24
\end{tabular}

Values are presented as mean \pm standard deviation or number. ASIA: American Spinal Injury Association.

TABLE 2: Results of ABPM in patients with spinal cord injury.

\begin{tabular}{lccc}
\hline & Daytime & Nighttime & $24 \mathrm{~h}$ \\
\hline SBP & $119.9 \pm 18.8$ & $123.8 \pm 21.2$ & $120.6 \pm 18.9$ \\
DBP & $75.6 \pm 11.0$ & $77.4 \pm 11.1$ & $75.8 \pm 10.7$ \\
HR & $76.3 \pm 14.1$ & $71.2 \pm 13.1^{*}$ & $75.1 \pm 13.4$ \\
AD event & $2.7 \pm 1.9$ & $3.1 \pm 4.8$ & $5.8 \pm 4.7$ \\
\hline
\end{tabular}

Values are presented as mean \pm standard deviation or number. ${ }^{*} p<0.05$, significant difference between the daytime values and the nighttime values. SBP: systolic blood pressure; DBP: diastolic blood pressure; HR: heart rate; $\mathrm{AD}$ : autonomic dysreflexia.

$100 \%$ ), and duration of SCI (32.6 versus 3.25). The percentage of cervical SCI was $83.3 \%$ versus $100 \%$ between AD and non$\mathrm{AD}$ groups. The percentage of motor complete and motor incomplete SCI was $12.5 \%$ versus $25 \%$ between AD and nonAD groups.

3.3. Comparative Results by Duration of Disease. In the subacute group, SBP and DBP were $119.5 \pm 19.4 \mathrm{mmHg}$ and $74.4 \pm 9.7 \mathrm{mmHg}$, respectively, in the daytime and $126.7 \pm 22.8 \mathrm{mmHg}$ and $77.2 \pm 12.1 \mathrm{mmHg}$, respectively, in the nighttime. In the subacute group, nighttime SBP and DBP were higher than the corresponding daytime values, showing the loss of physiologic nocturnal dipping; however, in the chronic group, the absence of physiologic nocturnal dipping was not observed. In the subacute group, there was a statistically significant HR decrease in the nighttime at $66.4 \pm$ $8.9 \mathrm{bpm}$ compared with that in the daytime at $74.0 \pm 12.3 \mathrm{bpm}$ $(p<0.01)$; however, in the chronic group, there was no statistically significant difference in the HR between daytime and nighttime $(p>0.05)$.

The frequency of AD in the subacute group was $2.1 \pm 1.8$ times in the daytime and $3.6 \pm 6.3$ times in the nighttime, and the average frequency was $5.7 \pm 5.8$ times in 24 hours. In the chronic group, it was $3.4 \pm 1.9$ times in the daytime and
TABLE 3: Comparison of ABPM values in subacute and chronic SCI.

\begin{tabular}{cccc}
\hline & Daytime & Nighttime & $24 \mathrm{~h}$ \\
\hline $\begin{array}{c}\text { SBP } \\
\text { Subacute }\end{array}$ & $119.5 \pm 19.4$ & $126.7 \pm 22.8^{*}$ & $120.6 \pm 19.7$ \\
$\begin{array}{c}\text { Chronic } \\
\text { DBP }\end{array}$ & $120.3 \pm 18.9$ & $120.5 \pm 19.5$ & $120.4 \pm 18.7$ \\
$\quad$ & & \\
Subacute & $74.4 \pm 9.7$ & $77.2 \pm 12.1$ & $74.4 \pm 10.1$ \\
Chronic & $77.0 \pm 12.6$ & $77.6 \pm 10.2$ & $77.3 \pm 11.4$ \\
HR & & & \\
$\begin{array}{l}\text { Subacute } \\
\text { Chronic }\end{array}$ & $74.0 \pm 12.3$ & $66.4 \pm 8.9^{* *}$ & $72.4 \pm 11.1$ \\
AD event & $78.8 \pm 16.1$ & $76.8 \pm 15.2^{\dagger}$ & $78.1 \pm 15.5$ \\
Subacute & $2.1 \pm 1.8$ & $3.6 \pm 6.3$ & \\
Chronic & $3.4 \pm 1.9$ & $2.6 \pm 2.2$ & $6.7 \pm 5.8$ \\
\hline
\end{tabular}

Values are presented as mean \pm standard deviation or number. ${ }^{*} p<0.05$ and ${ }^{* *} p<0.01$, significant difference between the daytime values and the nighttime values. ${ }^{\dagger} p<0.05$, significant difference between subacute and chronic SCI. SBP: systolic blood pressure; DBP: diastolic blood pressure; HR: heart rate; $\mathrm{AD}$ : autonomic dysreflexia.

$2.6 \pm 2.2$ times in the nighttime, and the average $\mathrm{AD}$ frequency was $6.0 \pm 3.3$ times in 24 hours (Table 3).

3.4. Comparative Results by the Severity of Injury. In the motor complete group, SBP and DBP were $102.5 \pm 14.2 \mathrm{mmHg}$ and $69.5 \pm 13.2 \mathrm{mmHg}$, respectively, in the daytime and $103.50 \pm 7.3 \mathrm{mmHg}$ and $68.25 \pm 6.1 \mathrm{mmHg}$, respectively, in the nighttime. In the motor incomplete group, SBP and DBP were $122.7 \pm 18.1 \mathrm{mmHg}$ and $76.6 \pm 10.5 \mathrm{mmHg}$, respectively, in the daytime and $127.2 \pm 20.8 \mathrm{mmHg}$ and $78.9 \pm 11.0 \mathrm{mmHg}$, respectively, in the nighttime. The nighttime SBP and DBP were higher than the corresponding daytime values, revealing the absence of physiologic nocturnal dipping in both groups. In the intergroup comparison, it was found that daytime, nighttime, and 24-hour SBP in the motor complete group were significantly lower than those in the motor incomplete group $(p<0.05)$.

There were no significant differences between daytime and nighttime HR in both groups $(p>0.05)$.

The frequency of $\mathrm{AD}$ was $3.5 \pm 1.9$ times in the daytime, $2.5 \pm 3.0$ times in the nighttime, and $6.0 \pm 4.1$ times in 24 hours in the motor complete group. In the motor incomplete group, it was $2.5 \pm 1.8$ times in the daytime, $3.25 \pm 5.1$ times in the nighttime, and $5.8 \pm 4.9$ times in 24 hours (Table 4 ).

3.5. Result of ADFSCI Questionnaire. The total scores of ADFSCI questionnaire ranged from 0 to 83 points with an average of $21.1 \pm 29.6$ points. Sixteen $(57.1 \%)$ symptomatic patients had a total score of at least 1 point and twelve $(42.9 \%)$ asymptomatic patients had a total score of 0 points. General characteristics of $\mathrm{AD}$ and non-AD groups were age (53.3 versus 59.7$)$, male sex (69\% versus $86.7 \%)$, cause of SCI (trauma $=84.6 \%$ versus $60.0 \%$ ), and duration of SCI $(20.2$ versus 35.5$)$. The percentage of cervical SCI was $92.3 \%$ versus $80.0 \%$ between $\mathrm{AD}$ and non-AD groups. The percentage of 
TABLE 4: Comparison of ABPM values between motor complete and incomplete SCI.

\begin{tabular}{lccc}
\hline & Daytime & Nighttime & $24 \mathrm{~h}$ \\
\hline SBP & & & \\
$\quad$ Complete & $102.5 \pm 14.2$ & $103.5 \pm 7.3$ & $102.5 \pm 11.0$ \\
$\quad$ Incomplete & $122.7 \pm 18.1^{\dagger}$ & $127.2 \pm 20.8^{\dagger}$ & $123.5 \pm 18.3^{\dagger}$ \\
DBP & & & \\
$\quad$ Complete & $69.5 \pm 13.2$ & $68.2 \pm 6.1$ & $69.2 \pm 10.7$ \\
$\quad$ Incomplete & $76.6 \pm 10.5$ & $78.9 \pm 11.0$ & $76.8 \pm 10.5$ \\
HR & & & \\
$\quad$ Complete & $73.2 \pm 10.2$ & $72.7 \pm 9.5$ & $73.5 \pm 10.3$ \\
$\quad$ Incomplete & $76.7 \pm 14.7$ & $71.0 \pm 13.7$ & $75.3 \pm 14.0$ \\
AD event & & & \\
Complete & $3.5 \pm 1.9$ & $2.5 \pm 3.0$ & $6.0 \pm 4.1$ \\
Incomplete & $2.5 \pm 1.8$ & $3.25 \pm 5.1$ & $5.8 \pm 4.9$ \\
\hline
\end{tabular}

Values are presented as mean \pm standard deviation or number. ${ }^{*} p<0.01$, significant difference between the daytime values and the nighttime values. ${ }^{\dagger} p<0.05$, significant difference between motor complete and incomplete SCI. SBP: systolic blood pressure; DBP: diastolic blood pressure; HR: heart rate; $\mathrm{AD}$ : autonomic dysreflexia.

motor complete SCI was $23.1 \%$ versus $6.7 \%$ between $\mathrm{AD}$ and non-AD groups.

3.6. Comparison of ABPM Results Depending on the Presence or Absence of Symptoms. In the symptomatic group, SBP and DBP were $124.7 \pm 25.2 \mathrm{mmHg}$ and $76.6 \pm 13.3 \mathrm{mmHg}$, respectively, in the daytime and $131.2 \pm 26.6 \mathrm{mmHg}$ and $80.3 \pm 14.6 \mathrm{mmHg}$, respectively, in the nighttime. SBP and DBP were higher at nighttime with a loss of the physiologic nocturnal dipping. In the asymptomatic group, SBP and DBP were $123.1 \pm 13.8 \mathrm{mmHg}$ and $77.9 \pm 12.2 \mathrm{mmHg}$, respectively, in the daytime and $122.1 \pm 19.9 \mathrm{mmHg}$ and $75.9 \pm 10.1 \mathrm{mmHg}$, respectively, in the nighttime. The loss of the physiologic nocturnal dipping was not observed in the asymptomatic group. In the intergroup comparison, daytime, nighttime, and 24-hour SBP tended to be higher in the symptomatic group than in the asymptomatic group, although no statistical significance was observed $(p>0.05)$.

No significant difference in HR was observed between daytime and nighttime in both groups $(p>0.05)$. The frequency of $\mathrm{AD}$ was $2.7 \pm 2.0$ times in the daytime, $3.0 \pm 2.9$ times in the nighttime, and $5.7 \pm 3.5$ times in 24 hours in the symptomatic group and was $3.3 \pm 1.7$ times in the daytime, $2.7 \pm 3.0$ times in the nighttime, and $6.0 \pm 3.3$ times in 24 hours in the asymptomatic group (Table 5 ).

\section{Discussion}

Autonomic nervous system disorders after SCI are common, among which $\mathrm{AD}$ and $\mathrm{OH}$ mainly occur due to the malfunction of the cardiovascular system. Cardiovascular complications have been reported to be the most common cause of death in patients with SCI, and the proposed causes include not only the metabolic syndrome, which consists of obesity, lipid metabolic disorders, and diabetes, but also a reduction of daily energy consumption due to reduced
TABLE 5: Comparison of ABPM values between symptomatic and asymptomatic $\mathrm{AD}$.

\begin{tabular}{lccc}
\hline & Daytime & Nighttime & $24 \mathrm{~h}$ \\
\hline SBP & & & \\
$\quad$ Symptomatic & $124.7 \pm 25.2$ & $131.2 \pm 26.6$ & $126.7 \pm 25.1$ \\
$\quad$ Asymptomatic & $123.1 \pm 13.8$ & $122.1 \pm 19.9$ & $122.5 \pm 14.5$ \\
DBP & & & \\
$\quad$ Symptomatic & $76.6 \pm 13.3$ & $80.3 \pm 14.6$ & $77.7 \pm 13.2$ \\
$\quad$ Asymptomatic & $77.9 \pm 12.2$ & $75.9 \pm 10.1$ & $77.1 \pm 11.3$ \\
HR & & & \\
Symptomatic & $78.8 \pm 14.7$ & $74.2 \pm 14.4$ & $77.6 \pm 14.4$ \\
Asymptomatic & $75.9 \pm 15.2$ & $69.7 \pm 14.8$ & $74.5 \pm 14.4$ \\
AD event & & & \\
$\quad$ Symptomatic & $2.7 \pm 2.0$ & $3.0 \pm 2.9$ & $5.7 \pm 3.5$ \\
Asymptomatic & $3.3 \pm 1.7$ & $2.7 \pm 3.0$ & $6.0 \pm 3.3$ \\
\hline
\end{tabular}

Values are presented as mean \pm standard deviation or number. SBP: systolic blood pressure; $\mathrm{DBP}$ : diastolic blood pressure; HR: heart rate; $\mathrm{AD}$ : autonomic dysreflexia.

physical activity [14-16]. In addition, a high prevalence of cardiovascular disorders, such as abnormal BP control and arrhythmias, and abnormal cardiovascular responses after exercise have also been suggested as important causes [11]. Despite the high prevalence of abnormal BP control in patients with SCI, currently, evaluations are only restricted to symptoms and signs that appear in each individual patient with SCI at the time of hypotensive and hypertensive crises. Furthermore, there is no accurate report of $\mathrm{AD}$ and $\mathrm{OH}$ prevalence in South Korea, as determined using accurate assessment tools.

While the prevalence of AD in patients with SCI has been variously reported to range from 20 to $70 \%$, it is known to occur in more than $90 \%$ of patients with cervical or upper thoracic SCI $[17,18]$. In general, the diagnostic definition of $\mathrm{AD}$ is a rise in SBP by more than $20-40 \mathrm{mmHg}$ [2]. However, considering that the stabilized $\mathrm{BP}$ of patients with cervical or upper thoracic SCI is reported to be lower than that of healthy persons by $15-20 \mathrm{mmHg}[8,19]$, normal or slightly elevated $\mathrm{BP}$ can be diagnosed as AD. Thus, in this study, we intended to determine the prevalence rate of $\mathrm{AD}$ using $\mathrm{ABPM}$ and the ADFSCI questionnaire in patients with cervical and upper thoracic SCI. According to a previous study [13], ABPM is a useful test for assessing BP instability and fluctuation of $\mathrm{BP}$ in the daytime and nighttime in SCI patients with AD. According to the results of this study, the prevalence rate of $\mathrm{AD}$ in patients with cervical and upper thoracic SCI, which was determined using ABPM, was 92.8\%, and this result was consistent with the previous study. According to the results of the ADFSCI questionnaire in this study, AD prevalence was $57.1 \%$, which was similar to a previous study which showed that $\mathrm{AD}$ symptoms occurred in about $70 \%$ of patients [20].

This typical daily BP pattern in SCI patients is characterized by the occurrence of potentially life-threatening hypertensive events due to $\mathrm{AD}$ as well as hypotensive events [21]. These immense BP fluctuations could contribute to increased shear stress of blood vessels and eventually to 
vascular injury, predisposing this population to a greater risk of arterial disease [22]. Although the impact of AD on the prevalence of cardiovascular and cerebrovascular diseases is not fully clarified, considering the report that cardiovascular instability in patients with SCI increased the frequency of heart disease and ischemic stroke occurrence [23], appropriate $\mathrm{BP}$ control and evaluation of cardiovascular instability are important. In addition, inappropriate activation of the sympathetic nervous system, which is associated with $\mathrm{AD}$, occurs several times a day and could be asymptomatic in spite of the significantly elevated arterial BP $[9,24]$. Thus, it is necessary to diagnose such asymptomatic $\mathrm{AD}$ earlier on and apply appropriate treatment interventions. In this present study, although the risk of morbidity from heart disease or cerebral vascular disease in $35.7 \%$ of the patients who were diagnosed with asymptomatic $\mathrm{AD}$ is likely to increase significantly, it was difficult to diagnose such asymptomatic $\mathrm{AD}$ using onetime BP measurements. Therefore, it is suggested that, as a preventive measure for cardiovascular disease in patients with cervical and upper thoracic SCI, early diagnosis and therapeutic interventions for $\mathrm{AD}$ are required using $\mathrm{ABPM}$ in the early stage. Medical personnel, caregivers, and individuals with SCI should be aware of the importance of the timely diagnosis and management of this life-threatening condition, which can result in a variety of significant complications including stroke, seizures, myocardial ischemia, and death [25].

It has been reported that the prevalence of $\mathrm{AD}$ increases according to the level and severity of SCI [20, 26, 27]. A previous study reported that $\mathrm{AD}$ occurred in $91 \%$ of patients with complete quadriplegia and only in $27 \%$ of patients with incomplete injury [20]. Based on the results of ABPM, in this study, AD was observed in $100 \%$ and $91.6 \%$ of patients with motor complete and motor incomplete SCI, respectively. This differs from the previous report on the prevalence of $\mathrm{AD}$ in incomplete injury, because the previous report was about the prevalence of $\mathrm{AD}$ during the urodynamic study in chronic SCI patients. Moreover, in the intergroup comparison, significant differences in daytime, nighttime, and 24-hour SBP were observed, in which AD occurred more frequently in the motor complete group with a concomitant increase in the number of $\mathrm{AD}$ events.

The timing of $\mathrm{AD}$ onset is not yet clear. Although previous researchers believed that $\mathrm{AD}$ was only limited to chronic SCI, recent reports show AD occurrence one day or several weeks after SCI $[27,28]$. These results suggest that patients with acute and subacute SCI were not diagnosed early and did not receive appropriate treatment for AD. Moreover, the relationships between the level and severity of injury and the timing of $\mathrm{AD}$ onset are currently not clear.

The phenomenon where SBP and DBP decrease at nighttime by at least $10 \%$ compared with daytime values is defined as physiological nocturnal BP dipping [29] and is reportedly the most sensitive predictor of cardiovascular disorders [12]. In patients with SCI, the absence of this nocturnal BP dipping or even the elevation of nocturnal $\mathrm{BP}$ has been observed. According to the results of the ABPM analysis performed in this study, in which the patients were divided into subacute and chronic stages by setting 3 months as the cut-off of the disease onset, it was found that the nighttime SBP in the subacute group was higher by $7 \mathrm{mmHg}$ compared to the chronic group, and this result was consistent with a previous report of nocturnal BP dipping loss in patients with SCI [12]. The subacute group showed a significant difference in the daytime and nighttime SBP $(p<0.05)$ with a concomitant loss of nocturnal BP dipping and a significant decrease in the nighttime HR $(p<0.01)$. However, the chronic group showed no significant differences in nocturnal BP dipping and HR. Considering the report that significant morphological changes such as atrophy of sympathetic preganglionic neurons and loss of dendritic arbors were observed in the acute phase [30], it is believed that neural regenerationmediated recovery of the autonomic nervous system by passage of time or acclimatization of patients to the stimuli and symptoms, which cause $\mathrm{AD}$ and acquisition of selfcontrol ability [9], influenced the above results.

Limitations of this study were (1) the small sample size of the subjects, (2) the incomplete exclusion of causes that may induce $\mathrm{AD}$, and (3) the fact that although the relationship between activities and $\mathrm{AD}$ onset was analyzed by recording activities of daily living in the daytime, the application of this type of evaluation, which is based on the record of patients or caregivers, is limited due to its lack of accuracy. Therefore, additional research addressing these limitations is necessary.

\section{Conclusion}

The results of this study showed that the incidence rate of $\mathrm{AD}$ was $92.8 \%$ in patients with cervical and upper thoracic SCI and the incidence rate of asymptomatic AD was $42.9 \%$. To manage long-term cardiovascular diseases and to lower mortality in patients with SCI, aggressive diagnosis and therapeutic interventions for $\mathrm{AD}$ using $\mathrm{ABPM}$ in the early stage should be considered even in asymptomatic patients.

\section{Conflicts of Interest}

The authors have no financial conflicts of interest.

\section{Acknowledgments}

This paper was supported in part by Soongsan Fellowship in Wonkwang University in 2014.

\section{References}

[1] C. J. Mathias and H. L. Frankel, "Cardiovascular control in spinal man," Annual Review of Physiology, vol. 50, pp. 577-592, 1988.

[2] R. W. Teasell, J. M. O. Arnold, A. Krassioukov, and G. A. Delaney, "Cardiovascular consequences of loss of supraspinal control of the sympathetic nervous system after spinal cord injury," Archives of Physical Medicine and Rehabilitation, vol. 81, no. 4, pp. 506-516, 2000.

[3] G. M. Yarkony, R. T. Katz, and Y. C. Wu, "Seizures secondary to autonomic dysreflexia," Physical Medicine and Rehabilitation, vol. 67, pp. 834-835, 1986.

[4] Z. M. Pine, S. D. Miller, and J. A. Alonso, "Atrial fibrillation associated with autonomic dysreflexia," American Journal of 
Physical Medicine \& Rehabilitation, vol. 70, no. 5, pp. 271-273, 1991.

[5] I. Eltorai, R. Kim, M. Vulpe, H. Kasravi, and W. Ho, "Fatal cerebral hemorrhage due to autonomic dysreflexia in a tetraplegic patient: Case report and review," Paraplegia, vol. 30, no. 5, pp. 355-360, 1992.

[6] M. Vallès, J. Benito, E. Portell, and J. Vidal, "Cerebral hemorrhage due to autonomic dysreflexia in a spinal cord injury patient," Spinal Cord, vol. 43, no. 12, pp. 738-740, 2005.

[7] D. Dolinak and E. Balraj, "Autonomic dysreflexia and sudden death in people with traumatic spinal cord injury," The American Journal of Forensic Medicine and Pathology, vol. 28, no. 2, pp. 95-98, 2007.

[8] C. J. Mathias and R. Bannister, "Autonomic disturbances in spinal cord lesions," in Autonomic Failure: A Textbook of Clinical Disorders of the Autonomic Nervous System, D. J. Mathias, Ed., pp. 839-881, Oxford University Press, Oxford, UK, 2002.

[9] T. A. Linsenmeyer, D. I. Campagnolo, and I.-H. Chou, "Silent autonomic dysreflexia during voiding in men with spinal cord injuries," The Journal of Urology, vol. 155, no. 2, pp. 519-522, 1996.

[10] M. Ekland, A. Krassioukov, K. E. McBride, and S. L. Elliott, "Incidence of autonomic dysreflexia and silent autonomic dysreflexia in men with SCI undergoing sperm retrievel: implications for clinical practice," The Journal of Spinal Cord Medicine, vol. 30, pp. 43-50, 2007.

[11] J. Myers, M. Lee, and J. Kiratli, "Cardiovascular disease in spinal cord injury: an overview of prevalence, risk, evaluation, and management," American Journal of Physical Medicine \& Rehabilitation, vol. 86, no. 2, pp. 142-152, 2007.

[12] M. Hubli and A. V. Krassioukov, "Ambulatory blood pressure monitoring in spinal cord injury: Clinical practicability," Journal of Neurotrauma, vol. 31, no. 9, pp. 789-797, 2014.

[13] M. Hubli, C. M. Gee, and A. V. Krassioukov, "Refined assessment of blood pressure instability after spinal cord injury," American Journal of Hypertension, vol. 28, no. 2, pp. 173-181, 2015.

[14] H. Krum, L. G. Howes, D. J. Brown et al., "Risk factors for cardiovascular disease in chronic spinal cord injury patients," Paraplegia, vol. 30, no. 6, pp. 381-388, 1992.

[15] M. J. DeVivo, J. S. Krause, and D. P. Lammertse, "Recent trends in mortality and causes of death among persons with spinal cord injury," Archives of Physical Medicine and Rehabilitation, vol. 80, no. 11, pp. 1411-1419, 1999.

[16] E. Garshick, A. Kelley, S. A. Cohen et al., "A prospective assessment of mortality in chronic spinal cord injury," Spinal Cord, vol. 43, no. 7, pp. 408-416, 2005.

[17] R. L. Braddom and J. F. Rocco, "Autonomic dysreflexia. A survey of current treatment," American Journal of Physical Medicine \& Rehabilitation, vol. 70, no. 5, pp. 234-241, 1991.

[18] R. Lindan, E. Joiner, A. A. Freehafer, and C. Hazel, "Incidence and clinical features of autonomic dysreflexia in patients with spinal cord injury," Paraplegia, vol. 18, no. 5, pp. 285-292, 1980.

[19] V. E. Claydon, S. L. Elliott, A. W. Sheel, and A. Krassioukov, "Cardiovascular responses to vibrostimulation for sperm retrieval in men with spinal cord injury," The Journal of Spinal Cord Medicine, vol. 29, no. 3, pp. 207-216, 2006.

[20] A. Curt, B. Nitsche, B. Rodic, B. Schurch, and V. Dietz, "Assessment of autonomic dysreflexia in patients with spinal cord injury," Journal of Neurology, Neurosurgery \& Psychiatry, vol. 62 , no. 5, pp. 473-477, 1997.
[21] A. Krassioukov and V. E. Claydon, "The clinical problems in cardiovascular control following spinal cord injury: an overview," Progress in Brain Research, vol. 152, pp. 223-229, 2006.

[22] S. A. Steins, M. C. Johnson, and P. J. Lyman, "Cardiac rehabilitation in patients with spinal cord injury," Physical Medicine \& Rehabilitation Clinics of North America, vol. 6, pp. 263-296, 1995.

[23] J.-C. Wu, Y.-C. Chen, L. Liu et al., "Increased risk of stroke after spinal cord injury: A nationwide 4-year follow-up cohort study," Neurology, vol. 78, no. 14, pp. 1051-1057, 2012.

[24] S. C. Kirshblum, J. G. House, and K. C. O’Connor, "Silent autonomic dysreflexia during a routine bowel program in persons with traumatic spinal cord injury: A preliminary study," Archives of Physical Medicine and Rehabilitation, vol. 83, no. 12, pp. 1774-1776, 2002.

[25] D. Wan and A. V. Krassioukov, "Life-threatening outcomes associated with autonomic dysreflexia: A clinical review," The Journal of Spinal Cord Medicine, vol. 37, no. 1, pp. 2-10, 2014.

[26] C. J. Mathias and H. L. Frankel, "The cardiovascular system in tetraplegia and paraplegia," in Handbook of Clinical Neurology, H. L. Frankel, Ed., Elsevier Science, New York, NY, USA, 1992.

[27] A. V. Krassioukov, J. C. Furlan, and M. G. Fehlings, "Autonomic dysreflexia in acute spinal cord injury: an under-recognized clinical entity," Journal of Neurotrauma, vol. 20, no. 8, pp. 707716, 2003.

[28] J. R. Silver, "Early autonomic dysreflexia," Spinal Cord, vol. 38, no. 4, pp. 229-233, 2000.

[29] W. S. Seo and H. S. Oh, "The circadian rhythms of blood pressure and heart rate in the hypertensive subjects: Dippers and non-dippers," Yonsei Medical Journal, vol. 43, no. 3, pp. 320328, 2002.

[30] A. V. Krassioukov and L. C. Weaver, "Reflex and morphological changes in spinal preganglionic neurons after cord injury in rats," Clinical and Experimental Hypertension, vol. 17, no. 1-2, pp. 361-373, 1995. 


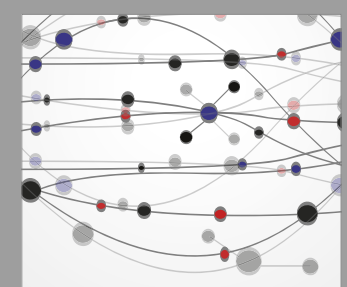

The Scientific World Journal
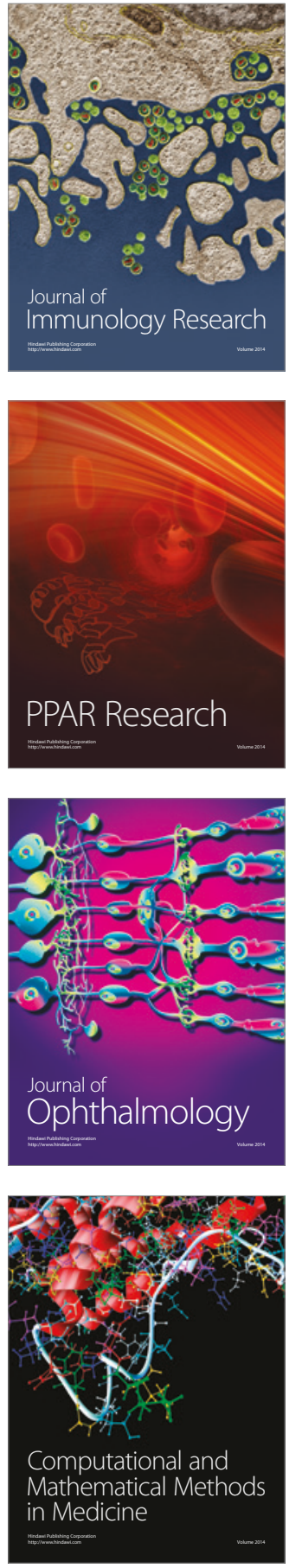

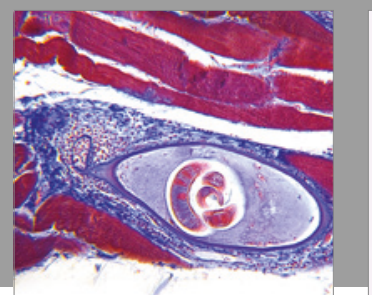

Gastroenterology Research and Practice
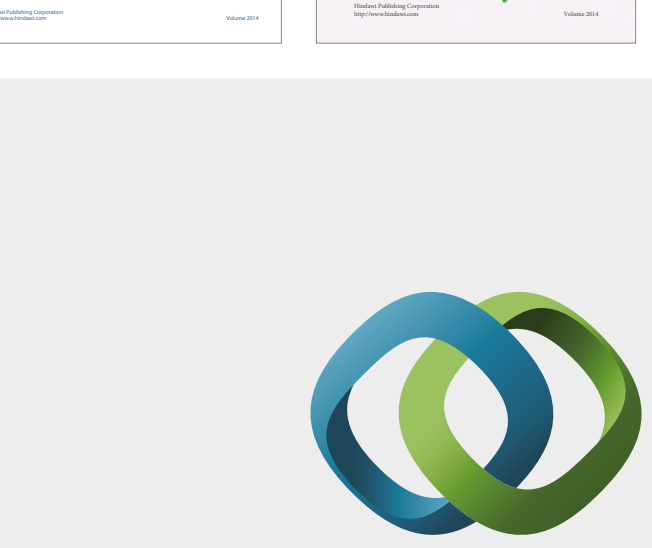

\section{Hindawi}

Submit your manuscripts at

https://www.hindawi.com
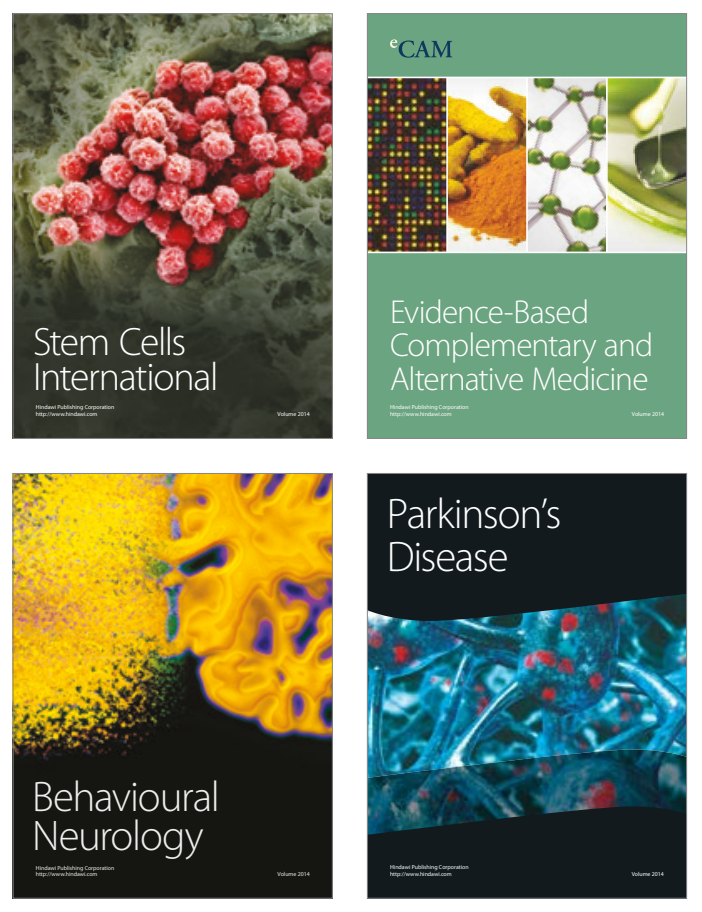
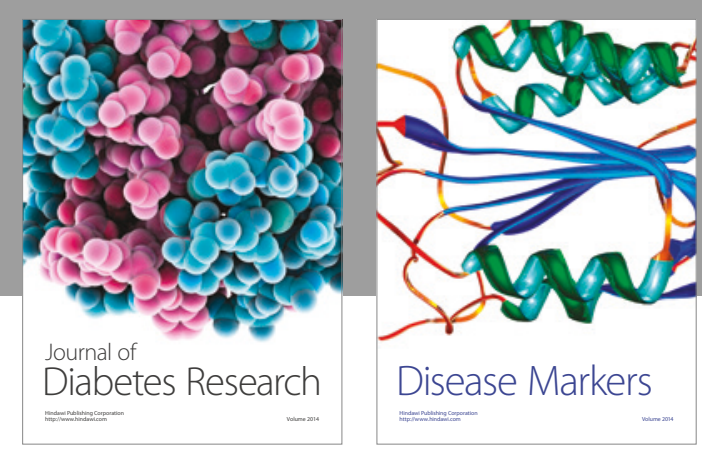

Disease Markers
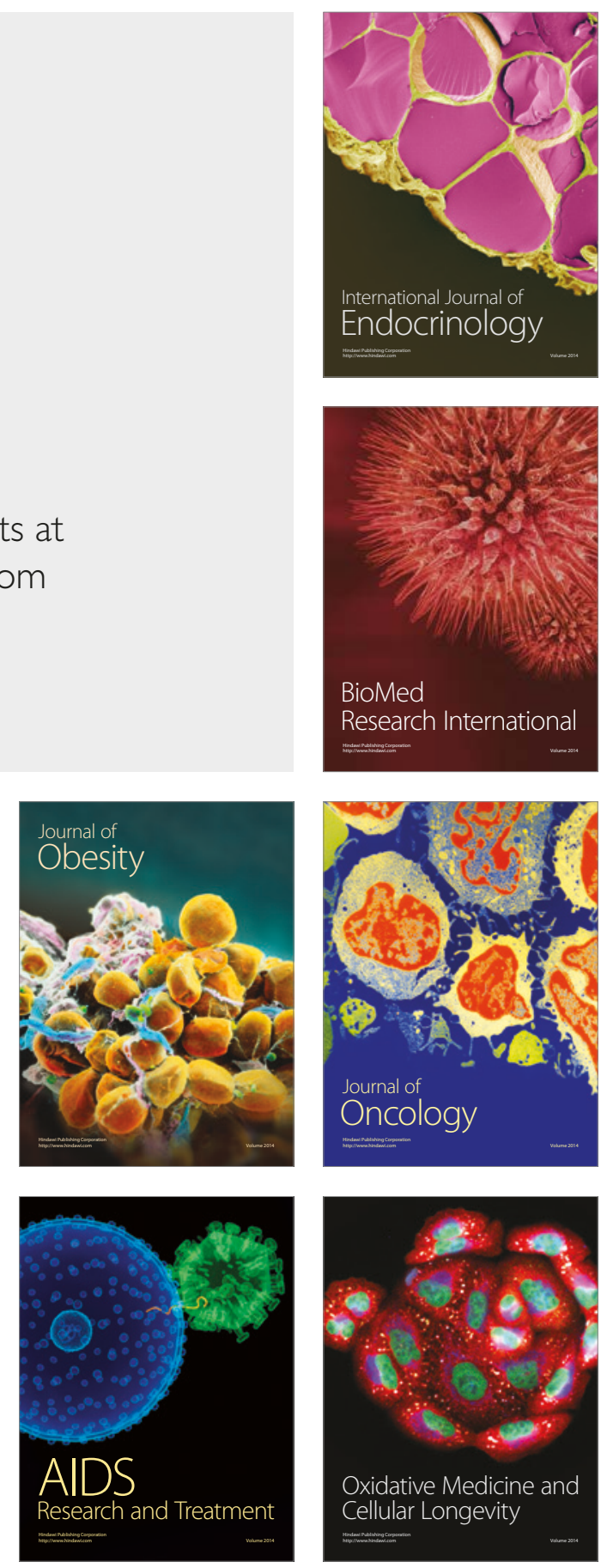